\title{
Dislocation Unbinding in Dense Two-Dimensional Crystals
}

\author{
Peter Bladon and Daan Frenkel \\ FOM-Institute for Atomic and Molecular Physics, Kruislaan 407, 1098 SJ Amsterdam, The Netherlands
}

(Received 14 November 1994)

\begin{abstract}
Two-dimensional solids of particles with short-range attraction exhibit a solid-solid critical point. We report computer simulations that show that, near this point, the crystal becomes unstable to dislocation unbinding. Since the concentration of free dislocations is very small the resulting phase should be a stable hexatic phase. As the range of the attraction grows, the hexatic pocket expands and moves towards the melting curve. Such hexatic phases near a solid-solid critical point should be experimentally observable in confined colloidal suspensions.

PACS numbers: 64.70.Kb, 61.20.Ja, 64.60.Cn
\end{abstract}

The debate about the nature of the melting transition in (quasi-) two-dimensional systems dates back to the seminal work of Landau and Peierls, who showed that there is no long-range positional order in two-dimensional crystals (see, e.g., [1]). In the early seventies, Kosterlitz and Thouless suggested that melting in two dimensions might proceed via a continuous dislocation-unbinding transition [2]. Subsequently, Halperin and Nelson [3] argued that the phase that results after dislocation unbinding is not an isotropic liquid, as it still has quasi-long-range bondorientational order. A second (disclination-unbinding) transition is required to go from this bond-ordered phase, termed "hexatic" in Ref. [3], to the isotropic fluid. In the hexatic phase there is a rapid (exponential) decay of translational correlations but only a slow (algebraic) decay of correlations of the local crystal axis. The continuous dislocation-unbinding transition can only occur when the dimensionless combination of elastic constants $K$ equals $16 \pi$ :

$$
K=\frac{4 \mu(\mu+\lambda)}{2 \mu+\lambda}=16 \pi .
$$

The quantities $\lambda$ and $\mu$ are the Lamé elastic constants, rendered dimensionless through multiplication by $a_{0}^{2} / k_{B} T$, where $a_{0}$ is the lattice spacing. Young [4] has shown that, close to the melting transition, the elastic constant $K$ has a universal scaling behavior $K \sim t^{0.3696 \ldots}$. It should be noted that the Kosterlitz-Thouless-Halperin-NelsonYoung (KTHNY) theory only predicts the point at which the solid becomes unstable to a spontaneous generation of free dislocations. The theory does not exclude the possibility that a first-order melting transition to the isotropic fluid phase intercedes at a point where the solid is still stable with respect to dislocation unbinding (i.e., for $K>16 \pi$ ). Unfortunately, in most simulation studies, the point where $K$ reaches the value $16 \pi$ is depressingly close to the point where first-order melting seems to take place. In fact, extensive simulation studies of a variety of two-dimensional model systems have thus far failed to provide compelling evidence for the KTHNY melting scenario and some even come close to proving the opposite [5-7].

$0031-9007 / 95 / 74(13) / 2519(4) \$ 06.00$
An alternative possibility is that two-dimensional solids melt by a spontaneous proliferation of grain boundaries. Fisher, Halperin, and Morf [8] showed that the grainboundary melting should only be expected if $E_{c}$, the core energy of dislocations, is not large compared to $k_{B} T$. A more quantitative prediction was subsequently made by Chui [9], who argued that grain boundary proliferation is the preferred melting mechanism if $E_{c}$ is less than $2.84 k_{B} T$. Simulation of a defect Hamiltonian by Saito [10] confirmed this picture: for $E_{c}$ below $2.84 k_{B} T$, there is a first-order transition caused by a nucleation of grain-boundary loops; when $E_{c}$ exceeds $2.84 k_{B} T$, melting takes place via a continuous transition of the KosterlitzThouless type. It appears, then, that the defect core energy is the vital predictor of the melting mechanism. In the systems simulated to date, there has been no opportunity to systematically vary the defect core energy, and therefore no opportunity to explore the region of parameter space where the KTHNY theory should be valid (for a review, see, e.g., [5,6]).

In this Letter we present unambiguous numerical evidence for the existence of a Kosterlitz-Thouless dislocation-unbinding transition in a two-dimensional system with short-range attractive interactions. Contrary to expectation, we find regions of hexatic phase far from the melting line. The hexatic regions form due to the presence of a nearby critical point that terminates a line of first-order isostructural solid-solid coexistence. Such a solid-solid critical point in a two-dimensional crystal was recently demonstrated by one of us [11]. In fact, the simulations in [11] showed that, both in two and in three dimensions, solids with sufficiently short-range attractive potentials can exist in a "high-" and a "low-" density solid phase. These phases have the same structure and are separated by a line of first-order phase transitions that terminate in a critical point. Figure 1 shows the generic form of the high-density phase diagram of a system with short-range attractive forces that undergoes an isostructural solid-solid transition. The precise shape of the phase diagram depends on the width of the attractive well. For three-dimensional systems, such solid-solid 


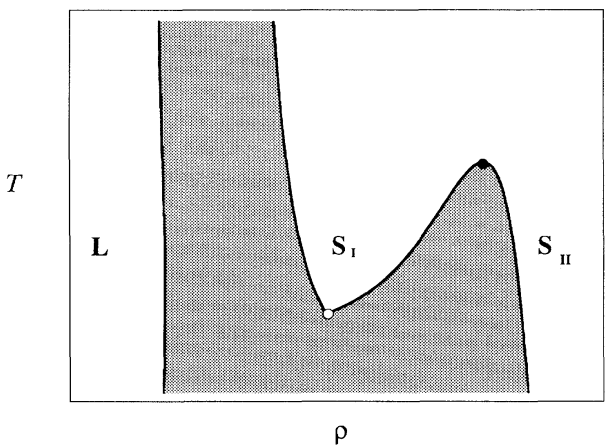

FIG. 1. The generic phase diagram for the isostructural solid-solid transition in the $(\rho, T)$ plane. Low-density solid $S_{I}$ coexists with a higher density solid $S_{I I}$, separated by a critical point (O). The triple point (O) marks the point of $S_{I}, S_{I I}$, and liquid $L$ coexistence. Regions of two-phase coexistence are shaded grey.

transitions have subsequently been analyzed theoretically by several authors $[12,13]$. However, as we argue below, the situation in two dimensions is more subtle because the solid-solid critical point will "induce" a hexatic phase. The reason why this should be so can be understood by considering the equation for $K$ [see Eq. (1)]. In two dimensions the bulk modulus equals $B=\lambda+\mu$ (we use the symbol $B$ for the bulk modulus to avoid confusion with the Kosterlitz-Thouless elastic constant $K$ ). Well away from the critical point $B$ is relatively large. Close to the critical point, where $B$ vanishes,

$$
K=\frac{4 \mu B}{\mu+B} \sim 4 B\left(1-\frac{B}{\mu}+\cdots\right),
$$

can be made arbitrarily small. Note that the shear modulus $\mu$ is not strongly affected by a solid-solid critical point. Hence, there will be a finite region around the critical point where $K<16 \pi$ and the solid will, necessarily, become unstable with respect to dislocation unbinding.

In the computer simulations discussed below, we have considered the phase behavior of a simple system with short-range attractive forces, viz., the square-well model. The pair potential in this model is given by

$$
v(r)=\left\{\begin{array}{cc}
\infty, & 0 \leq r<\sigma, \\
-\epsilon, & \sigma \leq r<\sigma+\delta, \\
0, & r \geq \sigma+\delta,
\end{array}\right.
$$

where the particle diameter is $\sigma$, the well depth $\epsilon$, and the well width $\delta$. Henceforth we measure all temperatures in units of $\epsilon / k_{B}$ and set the particle diameter to one. The simulations of Ref. [11] showed that solidsolid coexistence is possible if the width $\delta$ of the square well is less than $\sim 7$ of the particle diameter. For longer-range attraction the triple-point temperature becomes greater than the critical-point temperature, and the low density solid disappears. For decreasing $\delta$ the solid-solid critical point moves to higher densities, while the ratio of the triple-point temperature to the critical temperature decreases. In the limit $\delta \rightarrow 0$, the critical density approaches the density of regular close packing.

In order to map out the regions in the phase diagram that are unstable to dislocation unbinding, we performed extensive molecular dynamics (MD) simulations of the two-dimensional square-well system in the vicinity of the solid-solid critical point. As our primary aim was to compute the elastic constants, most simulations were performed on a relatively small system of 224 particles in a box of size ratio $14: 8 \sqrt{3}$. Periodic boundary conditions were applied and constant temperature was maintained. Two well widths were chosen, $\delta=0.03$ and 0.06. The pressure tensor $\mathcal{P}$ was measured as a function of the reduced density $\rho$ and temperature $T$ on a grid surrounding the critical point. 'The reduced density is defined by $\rho=N \sigma^{2} / A$, where $A$ is the system area. Densities were varied in increments of 0.0025 , temperatures in units of 0.01 . The bulk modulus was determined by fitting the pressure $P=P(\rho, T)$ to a convenient analytic form [14] and using the relation $B=$ $\rho \partial P / \partial \rho$. Sheared-box simulations were used to measure $\mu$ for a similar (though coarser) grid of points in the $(\rho, T)$ plane. Densities were varied in increments of 0.005 , temperatures in units of 0.01 . The pressure tensor measured at a finite value of strain is related to the firstorder isothermal elastic constants $C_{i j}^{(1)}$ at zero strain by [15]

$$
C^{(1)}=-\left(A^{\prime} / A\right)(1+\varepsilon)^{-1} \mathcal{P}(1+\tilde{\varepsilon})^{-1},
$$

where $\varepsilon$ is the matrix of deformations and $\tilde{\varepsilon}$ denotes its transpose. The Lamé coefficient $\mu=C_{x y x y}$ is then obtained by a linear fit of $C_{x y}^{(1)}$ vs $\varepsilon_{x y}$, the applied shear strain. Care was taken to ensure that no shear flow occurred during the simulations and that the shear applied was sufficiently small to ensure a linear response of the off-diagonal stress tensor elements to the applied shear strain. The shear strain applied in any simulation was never more than $0.5 \%$. In practice it is more important to obtain an accurate estimate of the bulk modulus in the critical region, as far from the melting line the shear modulus remains high, and increases monotonically with density. Each simulation consisted, after equilibration, of $\sim 30000$ collisions per particle. Having obtained $B$ and $\mu$ as a function of density and temperature, it is a simple matter to obtain $K$ and delineate the regions of the phase diagram where dislocation unbinding should occur [see $\mathrm{Eq}(1)]$. The critical temperature and density were found to be $T_{c}=0.885, \rho_{c}=1.010$ and $T_{c}=0.895, \rho_{c}=1.046$ for the two studied well widths $\delta=0.03$ and $\delta=0.06$, respectively.

For such small systems at high density the defect density in our simulations was always effectively zero. This greatly facilitated the numerical calculations as the 
simulations could be relatively short, since there was no need to equilibrate defect structures. Even for a system of 16184 particles with $\delta=0.06$, simulations showed that, at the critical point, the density of unbound dislocations is negligible. The low concentration of defects is, in fact, the prime feature that makes the present model a suitable candidate for exhibiting a true dislocationunbinding transition, as it indicates the dislocation core energy must be very large. In fact by decreasing $\delta$ and moving the solid-solid transition to higher densities the core energy $E_{c}$ can be made arbitrarily large.

As there are, in practice, no defects in the system studied by simulation, the elastic constants that we measure are the "bare" or "unrenormalized" elastic constants of the Kosterlitz-Thouless theory. However, these represent an upper bound to the true, renormalized elastic constants of the infinite system. First, increasing the system size will reduce the elastic constants measured for the 224 particle system; longer wavelength phonons will "soften" the system. This effect can easily be observed by measuring the pressure along an isotherm of a system of twice the linear extent of the 224 particle system. Second, and more importantly, the presence of defects always renormalizes $K$ downwards. This is particularly obvious in that part of the phase diagram where we find $K$ to be less than $16 \pi$. In an infinite system, such values of $K$ are renormalized to zero. Hence, the range of stability of the hexatic phase will be larger than follows from the present simulations.

Figure 2 shows the phase diagram for $\delta=0.03$. The phase diagram for this well width has been reproduced from the data of [11]. Our data have been scaled so that the critical points occur at the same density and temperature. The region of solid unstable to dislocation unbinding has been shaded black, and is localized to the region immediately surrounding the critical point. Figure 3 shows the phase diagram for $\delta=0.06$. Here

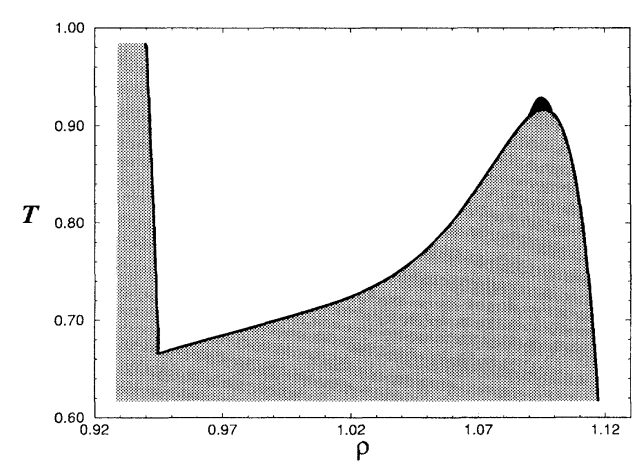

FIG. 2. The phase diagram for the 2D square-well system with $\delta=0.03$. The region of unstable solid around the solid-solid critical point - the hexatic region-is shaded black. Regions of two-phase coexistence are shaded grey. The density and temperature are expressed in units $\sigma^{-2}$ and $\epsilon / k_{B}$, respectively. At this high density, the critical point is far from the melting line confining the hexatic region to a small area around the critical point.

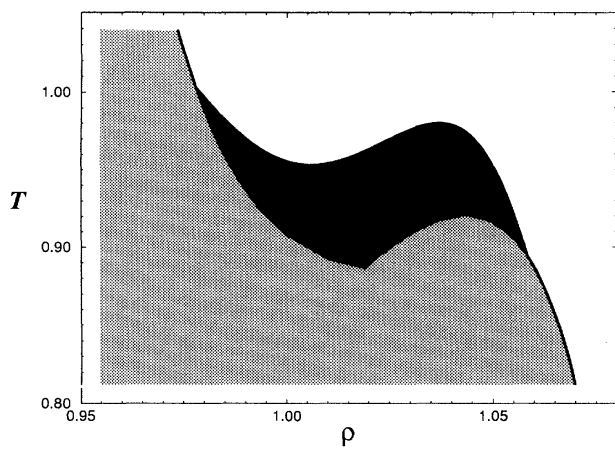

FIG. 3. The phase diagram for the 2D square-well system with $\delta=0.06$. The region of unstable solid around the solid-solid critical point - the hexatic region-is shaded black. Regions of two-phase coexistence are shaded grey. Units as in Fig. 2. At this value of $\delta$ the critical temperature is close to the triple-point temperature, causing the hexatic region to extend as far as the melting line.

the critical point is much closer to the melting line, and the triple point temperature is much closer to the critical temperature. The lower critical density for $\delta=0.06$ causes the bulk modulus to be a much more slowly increasing function of density than is the case for $\delta=$ 0.03 . The system is softer and the region of unstable solid extends over a much larger region around the critical point. The effect of the approaching melting line can clearly be seen. To the left of the critical point, the bulk modulus is approximately constant when compared to the rapid decrease of the shear modulus with decreasing density. This lowers $K$ towards unstable values as the melting curve is approached. It would be premature to conclude that the hexatic in this region melts via a disclination unbinding mechanism, as it is quite possible that the hexatic phase undergoes a first-order transition to the isotropic fluid.

In summary, we have shown that a $2 \mathrm{D}$ solid of particles with short-range attraction can be unstable to dislocation unbinding in a region that is clearly thermodynamically stable with respect to the isotropic fluid. We stress that our results should not be particularly sensitive to the form of the potential: Any sufficiently short-range potential is expected to induce a solid-solid transition [11,12], and hence a finite region of hexatic phase in the neighborhood of the critical point. The hexatic phase is formed due to density fluctuations occurring in the neighborhood of the solid-solid critical point, rather than long wavelength shear modes induced by the presence of the liquid-solid melting line. The regions of hexatic phase increase in extent as the range of the potential is increased, and sufficiently close to the $\delta$ value where the low-density solid phase becomes unstable, hexatic regions can extend as far as the melting line. The high density of the hexatic regions investigated results in a very low defect density. This prevents direct observation of the power-law decay of bond orientational correlation functions in the present simulations. 
However, the phase behavior described in this Letter should be experimentally observable in quasitwo-dimensional systems, such as colloids between glass plates. Such experimental systems should be large enough to reveal the algebraic decay of bond-orientational order. There are several ways to make colloids interact through an effective potential that has a deep and narrow attractive minimum, for instance, by adding small, nonadsorbing polymers. It should be noted that, even if the attractive well is too wide to induce a critical point in the solid phase, the vicinity of a critical point in the metastable solid should enhance the tendency towards dislocation unbinding in the stable solid. In this context, it is interesting to note that recent evidence suggests that, also in confined charge-stabilized colloids, the secondary minimum in the potential may be narrow and deep [16]. It is tempting to speculate that this short-range attraction between charge-stabilized colloids facilitated the formation of a hexatic phase in the quasi-two-dimensional system of polystyrene spheres studied by Murray and van Winkle (for a review, see [17]).

This work is part of the research program of the Foundation for Fundamental Research of Matter (FOM) and is supported financially by the Netherlands Organization for Scientific Research (NWO). P. B. acknowledges NATO/EPSRC for support and the FOM-Institute for its hospitality. We thank David Nelson, Thierry Biben, and Joost Frenken for a critical reading of the manuscript.

[1] R. Peierls, Surprises in Theoretical Physics (Princeton University Press, Princeton, 1979).

[2] J. M. Kosterlitz and D. J. Thouless, J. Phys. C 5, L124 (1972); J. Phys. C 6, 1181 (1973).
[3] B. I. Halperin and D. R. Nelson, Phys. Rev. Lett. 41, 121 (1978); D. R. Nelson and B. I. Halperin, Phys. Rev. B 19, 2457 (1979).

[4] A. P. Young, Phys. Rev. B 19, 1855 (1979).

[5] For a recent review, see M. A. Glaser and N. A. Clark, Adv. Chem. Phys. 83, 543 (1993), and references therein.

[6] Bond-Orientational Order in Condensed Matter Systems, edited by K. J. Strandburg (Springer, New York, 1992).

[7] B. J. Alder and T.E. Wainwright, Phys. Rev. 127, 359 (1962); J. A. Zollweg and G. V. Chester, Phys. Rev. B 46, 11186 (1992); J. Lee and K. J. Strandburg, ibid. 46, 11190 (1992); H. Weber and D. Marx, Europhys. Lett. 27, 593 (1994).

[8] D. S. Fisher, B. I. Halperin, and R. Morf, Phys. Rev. B 20, 4692 (1979).

[9] S. T. Chui, Phys. Rev. B 28, 178 (1983).

[10] Y. Saito, Phys. Rev. B 26, 6239 (1982).

[11] P. Bolhuis and D. Frenkel, Phys. Rev. Lett. 72, 2211 (1994); P. Bolhuis, M. Hagen, and D. Frenkel, Phys. Rev. E 50, 4880 (1994).

[12] A. Daanoun, C.F. Tejero, and M. Baus, Phys. Rev. Lett. 73, 752 (1994); A. Daanoun, C. F. Tejero, H. N.W. Lekkerkerker, and M. Baus, Phys. Rev. E 50, 2913 (1994).

[13] C. N. Likos, Zs. T. Németh, and H. Löwen, J. Phys. Condens. Matter 6, 10965 (1994).

[14] B. Mulder and D. Frenkel, Mol. Phys. 55, 1193 (1985).

[15] See, e.g., D. Frenkel, in Simple Molecular Systems at Very High Density, edited by A. Polian, P. Loubeyre, and N. Boccara (Plenum, New York, 1988).

[16] G. M. Kepler and S. Fraden, Phys. Rev. Lett. 73, 356 (1994).

[17] See the article by C.A. Murray in Bond-Orientational Order in Condense Matter Systems, Ref. [6]. 